\title{
TECNOLOGÍA PREVIA A LA RESTAURACIÓN DE EDIFICIOS HISTÓRICOS
}

\author{
(TECHNOLOGY PREVIOUS TO THE RESTAURATION OF HISTORICAL BUILDINGS)
}

Francisco Jurado Jiménez*, Arquitecto

Prof. del Dpto. de Estructuras de la Escuela Superior de Arquitectura de la U. P. M.

ESPAÑA

Fecha de recepción: $26-$ II -99

ESPANA

RESUMEN

En este artículo se trata de hacer una descripción de las herramientas y técnicas que se utilizan, o están disponibles, en todas las fases de trabajo anteriores a la propia restauración de un edificio histórico; desde la toma de datos inicial hasta la ejecución de la obra, pasando por la redacción del proyecto correspondiente.

\section{SUMMARY}

This article contains the description of the tools and techniques used, or those that are available, in all the phases of the work previous to the restauration of historical buildings, from initial data gathering until the execution of the work, once the project has been written.

\section{INTRODUCCIÓN}

Hablar de la tecnología en la restauración de edificios históricos tiene un doble peligro; por un lado, intentar convertirse en un abanderado que cante las excelencias de las nuevas herramientas, o bien, por otro, hacer una alabanza pormenorizada de los métodos tradicionales, como los más acordes con la intervención en edificios de cierta antigüedad.

Sin dejar de lado la parte útil de ambos extremos, hubiera sido muy sugerente titular este artículo como: "NUEVAS TÉCNICAS PARA VIEJAS CONSTRUCCIONES”, pero nuestra intención no es hacer descripciones exhaustivas de todas las posibilidades que hoy nos proporcionan mercado, sociedad y profesionales especialistas, sino hacer un viaje, a la luz de nuestra dilatada experiencia de más de veinte años realizando obras de restauración y conservación, sobre las técnicas que, en cada situación, en cada circunstancia, hemos podido utilizar. Al fin y al cabo, ésta es la más sincera realidad: la única tecnología realmente disponible es la que, a la postre, se acaba utilizando'.

En cualquier caso, el factor económico siempre será uno de los más determinantes $\mathrm{y}$, en muchos casos, el mayor o menor ingenio en los medios utilizados o en la solución adoptada va a depender, en modo inverso, de la cantidad de presupuesto disponible.

Para ordenar mínimamente este artículo, vamos a hablar de las técnicas, dividiéndolas en cuatro grandes grupos: los estudios previos, la monitorización, la realización del proyecto y la ejecución de la restauración ${ }^{2}$.

Los dos primeros apartados están, en muchos casos, incluidos en el tercero, es d vir, los pocos medios puestos a disposición de determinados encargos, hacen que sea directamente la realización del proyecto la primera de las fases. Es excesivamente frecuente que el propio redactor del proyecto no disponga de ningún estudio previo y que, si quiere hacer alguna monitorización, él mismo deba intentar convencer a alguien para poner un trozo de yeso que sirva como testigo de posibles movimientos en tal o cual fisura, cuando no es él mismo el que la marca con un lápiz para inspeccionar su posible progresión.

\section{LOS ESTUDIOS PREVIOS}

La importancia de los estudios previos es obvia cuando queremos conocer a fondo el edificio que vamos a restaurar y cuando queremos tener una diagnosis acértada de los problemas que padece e intentamos resolver.

\footnotetext{
(*) Todos los ejemplos citados en este artículo, corresponden a obras dirigidas por el autor.
} 
Podemos clasificarlos de distintas maneras; quizás la más clara sea aquélla que atiende a la distinta casuística que abordan.

\section{1.a) ESTUDIOSHISTÓRICOS}

En este apartado, poca es la tecnología que hemos tenido ocasión de conocer y emplear en los trabajos que hemos realizado. Sin embargo, no hay que desdeñar la gran importancia que pueden tener las investigaciones históricas, sobre todo atendiendo a los aspectos constructivos del edificio, para conocer la evolución que ha tenido a lo largo del tiempo.

En la evolución de los aspectos patológicos, quizás lo más interesante de nuestra experiencia haya sido, en las investigaciones previas a la restauración del acueducto romano de Segovia, la comparación entre fotografias de finales del siglo pasado y la situación actual, lo cual nos permitió observar el acelerado desgaste que los sillares graníticos han tenido en el último siglo, comparándolo con sus otros 18 siglos de vida anterior ${ }^{3}$.

\section{CLASIFICACIÓNDELOSESTUDIOS PREVIOS}

\begin{tabular}{|c|c|c|}
\hline 1.a) HISTÓRICOS & \multicolumn{2}{|l|}{$\begin{array}{l}\text { Documentales } \\
\text { Planimétricos } \\
\text { Grabados y Fotografias } \\
\end{array}$} \\
\hline 1.b) AMBIENTALES & \multicolumn{2}{|c|}{$\begin{array}{l}\text { Contaminación, higrotermia y otros } \\
\text { Estudios microbiológicos }\end{array}$} \\
\hline 1.c) GEOMÉTRICOS & \multicolumn{2}{|l|}{$\begin{array}{l}\text { Topografia } \\
\text { Fotogrametría } \\
\end{array}$} \\
\hline 1.d) ENSAYOS DE MATERIALES & \multicolumn{2}{|c|}{$\begin{array}{l}\text { De caracterización } \\
\text { Mecánicos } \\
\text { De comportamiento ante productos químicos }\end{array}$} \\
\hline 1.e) INVESTIGACIONES IN SITU & $\begin{array}{l}\text { Catas informativas } \\
\text { Catas arqueológicas } \\
\text { Catas y sondeos geotécnicos } \\
\text { Estudios geofisicos }\end{array}$ & $\begin{array}{l}\text { Geo-rádar } \\
\text { Resistividad eléctrica } \\
\text { Medición de vibraciones } \\
\text { Auscultación dinámica } \\
\text { Velocidad ultrasónica }\end{array}$ \\
\hline
\end{tabular}
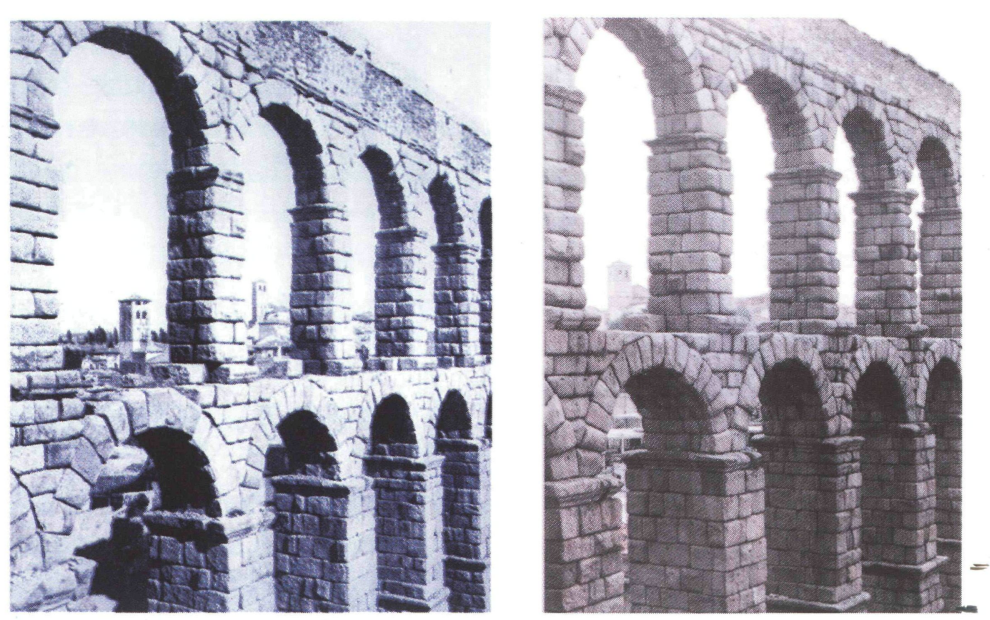

Fotografia del acueducto de Segovia en 1863 y la misma zona fotografiada en la actualidad. 


\section{3.d)MAQUETAS}

A pesar de la potente herramienta que supone el ordenador, hoy por hoy sigue siendo la maqueta la mejor manera de visualizar previamente el resultado volumétrico de una intervención. Desde la propia realización de maquetas totalmente artesanales de trabajo, con papel, "cartón pluma" o cualquier material de fácil corte, hasta las técnicas más profesionales utilizadas por los maquetistas especializados, que llegan a utilizar resinas y metales.

Aunque, también en este campo, el ordenador va introduciendo posibilidades cada vez más sofisticadas, desde el control de plotter de corte hasta el "tallado" completo de un volumen, transcrito directamente de su representación virtual informática.

\section{LA EJECUCIÓN DE LA RESTAURACIÓN}

Aunque ya se sale del contenido previsto en este artículo, puesto que lo haría excesivamente extenso, vamos a plantear una clasificación de las técnicas más habituales utilizadas en la propia obra.

De nuevo, sin ser exhaustivos, nos hemos limitado a citar los oficios y técnicas más habituales que podemos encontrarnos en el desarrollo de la propia obra de restauración ${ }^{15}$.

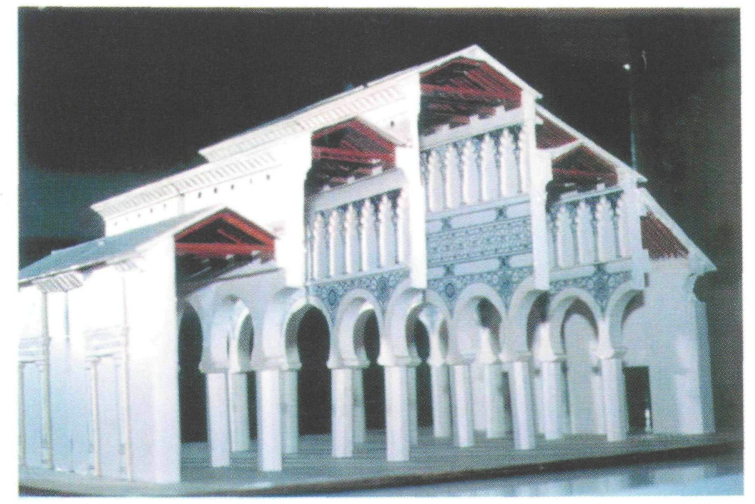

Maqueta de parte de la sinagoga de Santa Maria la Blanca, para un estudio sobre la solución de su cubierta.

Prácticamente cada uno de los apartados anteriores puede ser objeto de una publicación específica. De hecho, la bibliografia existente en nuestro país sobre distintos oficios es tan extensa que sobrepasa el objeto de este artículo su mera $\operatorname{cita}^{16}$.

Quizás, en una próxima ocasión podríamos desarrollar, de un modo más pormenorizado, cada uno de los puntos anteriores, siempre bajo nuestra punto de vista y nuestra experiencia en obras de restauración y conservación.

\section{CLASIFICACIÓN DE LAS TÉCNICASUTILIZADASENOBRAS DE} RESTAURACIÓN

\begin{tabular}{|c|c|c|}
\hline 4.a) RECALCES DE CIMENTACIONES & \multicolumn{2}{|l|}{$\begin{array}{l}\text { Micropilotaje } \\
\text { Jet grouting }\end{array}$} \\
\hline 4.b) LIMPIEZA DE PARAMENTOS & \multicolumn{2}{|c|}{$\begin{array}{l}\text { Manual, mecánica o química } \\
\text { Proyecciones de distintos micromateriales }\end{array}$} \\
\hline 4.c) INYECCIONES Y COSIDOS & \multicolumn{2}{|c|}{$\begin{array}{l}\text { Inyecciones de cal hidráulica, morteros especiales, resinas, etc. } \\
\text { Cosidos estáticos con aceros, fibras de vidrio, etc. } \\
\text { Tensados con aceros especiales }\end{array}$} \\
\hline 4.d) TÉCNICAS TRADICIONALES & \multicolumn{2}{|c|}{$\begin{array}{l}\text { Ladrillos y bóvedas tabicadas } \\
\text { Cantería } \\
\text { Cerámica, azulejería y vidriados } \\
\text { Vitralistas } \\
\text { Tapiales } \\
\text { Morteros de cal y agua de cal } \\
\text { Estucos y Revocos } \\
\text { Forja y metalistería } \\
\text { Carpintería y ebanistería } \\
\text { Pinturas especiales (pan de oro) y patinados (oxalatos) }\end{array}$} \\
\hline 4.e) MOLDEADOS Y REPRODUCCIONES & \multicolumn{2}{|c|}{$\begin{array}{l}\text { Moldes de silicona, poliéster, escayola, etc. } \\
\text { Prefabricados con piedra artificial, con resinas, etc. }\end{array}$} \\
\hline 4.f) MEDIOS AUXILIARES & $\begin{array}{l}\text { Instrumentos } \\
\text { Maquinaria }\end{array}$ & $\begin{array}{l}\text { Colorimetros } \\
\text { Pachómetros (detección de metales ocultos) } \\
\text { Esclerómetro (medida dureza superficial) } \\
\text { Grúas móviles } \\
\text { Compresores para hidrodemolición } \\
\text { Hilo de corte continuo }\end{array}$ \\
\hline
\end{tabular}


Modelos estructurales de una pila del acueducto de Segovia, con elementos finitos y con un programa de cálculo vectorial desarrollado para este fin.
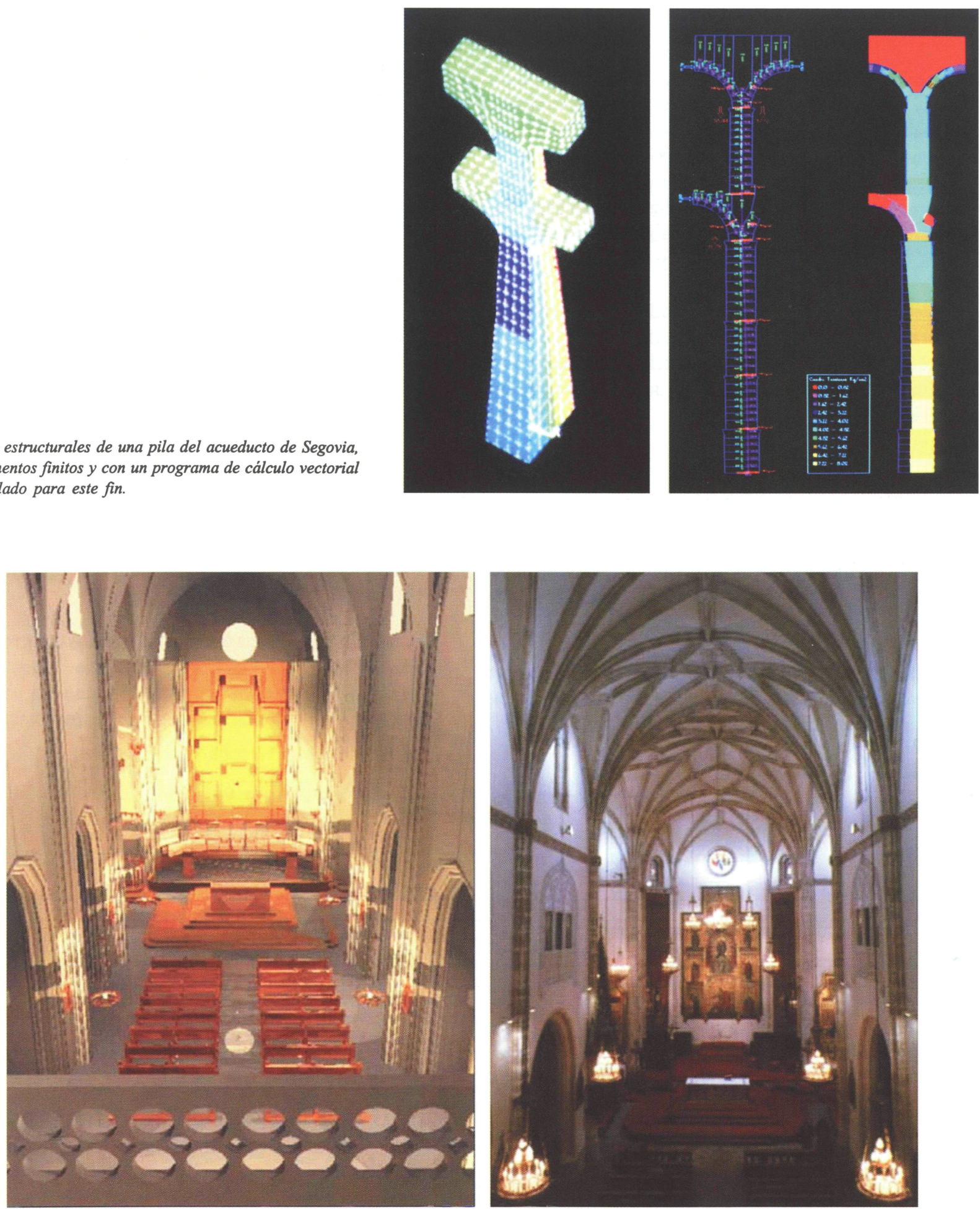

A la izquierda, simulación de la iluminación interior proyectada para el templo de San Jerónimo el Real de Madrid. A la derecha, el resultado real después de la intervención.

variable que pueda ser descrita con funciones matemáticas ${ }^{14}$. Las simulaciones de iluminación pueden llegar a resultar también muy útiles a la hora de intervenir en un edificio histórico. Pero, por supuesto, siempre será necesaria una calibración previa del modelo volumétrico

(c) Consejo Superior de Investigaciones Científicas

Licencia Creative Commons 3.0 España (by-nc) (en lo que respecta a materiales y su capacidad de reflexión) y de los distintos tipos de luz que se-introducen (respecto a su intensidad, temperatura de color y cono de luz) para ser conscientes de su parecido con la futura realidad. 


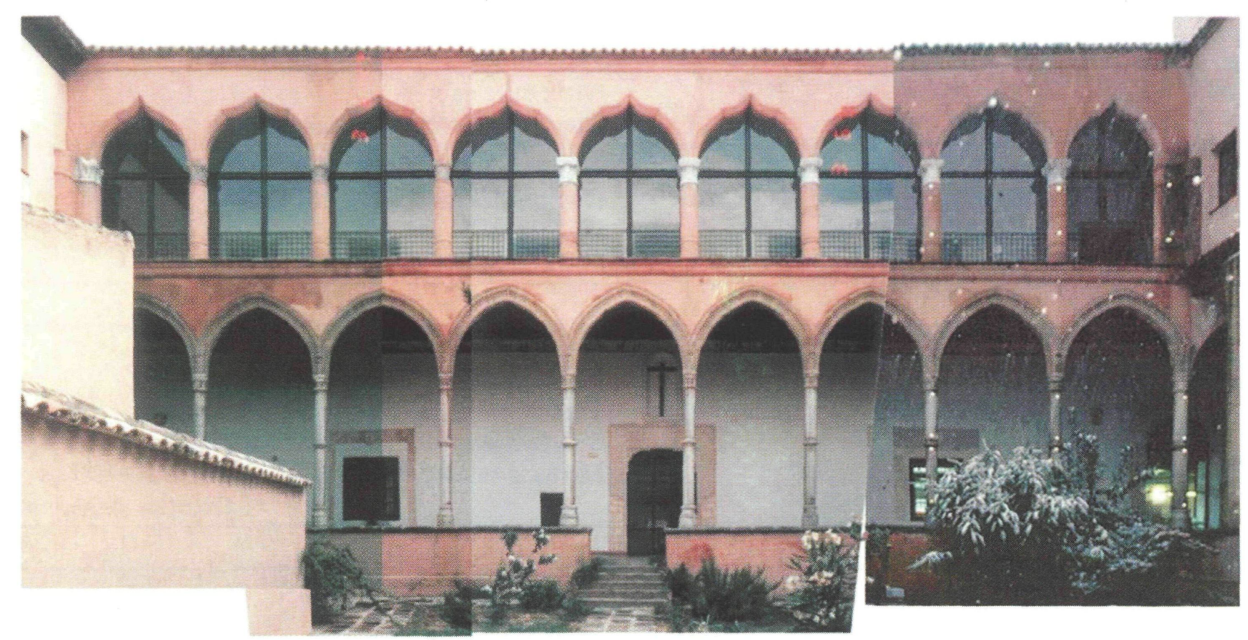

Fachada de un claustro de Toledo obtenida mediante ortogonalización y montaje de varias fotografias. El resultado es la fachada a escala (se conocian dos puntos de apoyo en cada columna).

\section{3.c) SIMULACIONESCONORDENADOR}

Dichos modelos volumétrícos asociados a montajes fotográficos pueden ser una simulación muy real del resultado previsto en una intervención sobre un conjunto edificado ${ }^{13}$.
Posiblemente sea la capacidad de realizar simulaciones la ventaja más aprovechable del ordenador. No hablamos solamente de recrear espacios arquitectónicos, sino de estudiar modelos de comportamiento estructural, realizar simulaciones de comportamiento térmico, de funcionamiento acústico, o de la evolución de cualquier
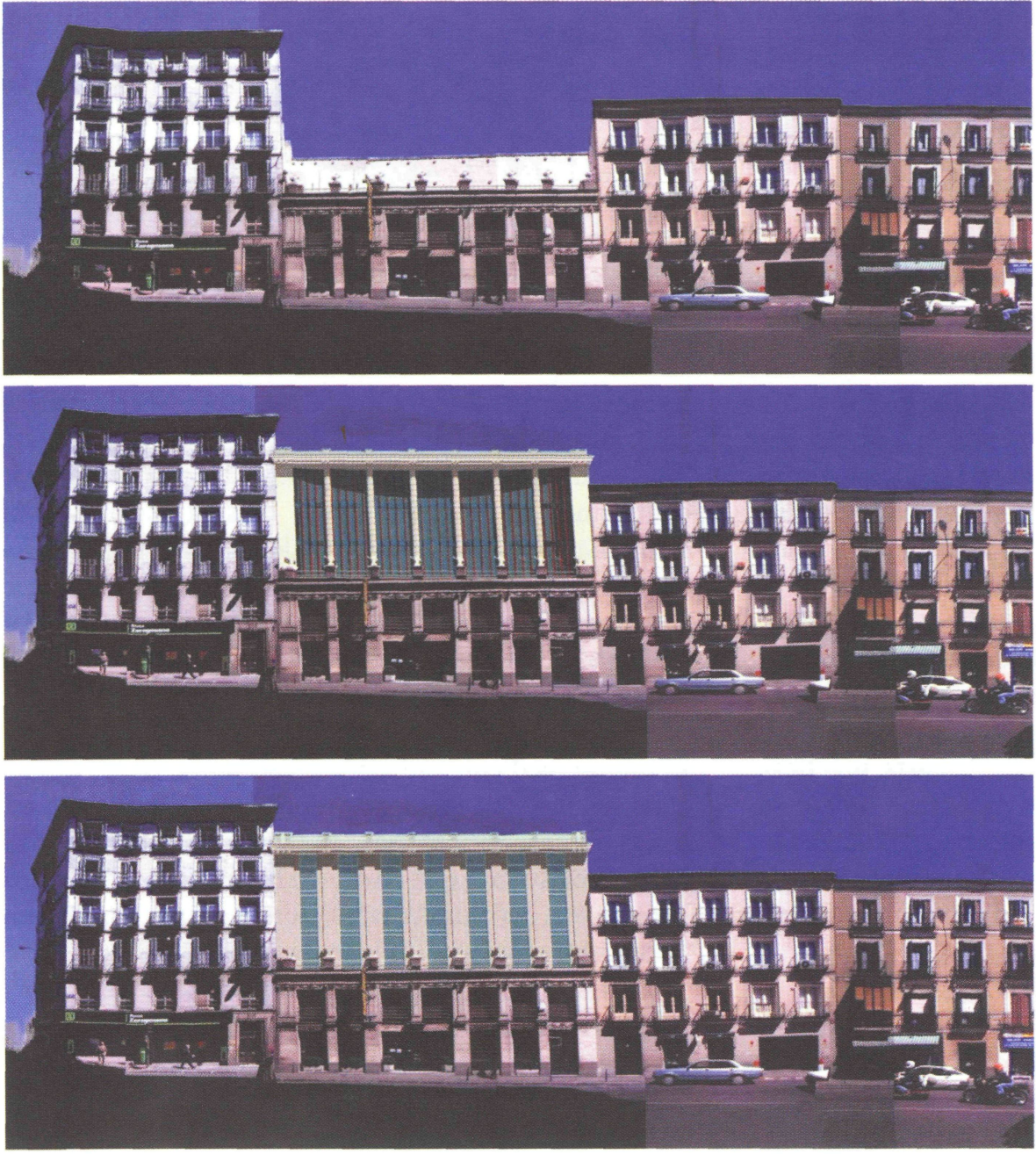

Montaje fotográfico que refleja el estado actual de una fachada protegida culturalmente en Madrid y simulaciones de dos propuestas de intervención sobre la misma. 
CLASIFICACIÓNDELATECNOLOGÍAUTILIZADAENLAPROYECTACIÓN

\begin{tabular}{|c|c|}
\hline 3.a) TOMA DE DATOS GEOMÉTRICOS & $\begin{array}{l}\text { Cinta métrica } \\
\text { Nivel, taquímetro y estación total }\end{array}$ \\
\hline 3.b) RESTITUCIONES Y REPRESENTACIÓN & $\begin{array}{l}\text { Ortogonalización fotográfica } \\
\text { CAD en } 2 \mathrm{D} \\
\mathrm{CAD} \text { en } 3 \mathrm{D}\end{array}$ \\
\hline 3.c) SIMULACIONES CON ORDENADOR & $\begin{array}{l}\text { Simulaciones volumétricas } \\
\text { Montajes fotorrealísticos } \\
\text { Simulaciones higro-térmicas } \\
\text { Simulaciones acústicas, de iluminación,... } \\
\begin{array}{ll}\text { Modelos estructurales } & \text { Elementos finitos } \\
& \text { Cálculos vectoriales }\end{array}\end{array}$ \\
\hline 3. d) MAQUETAS & $\begin{array}{l}\text { Artesanales } \\
\text { Plotter tridimensional }\end{array}$ \\
\hline
\end{tabular}
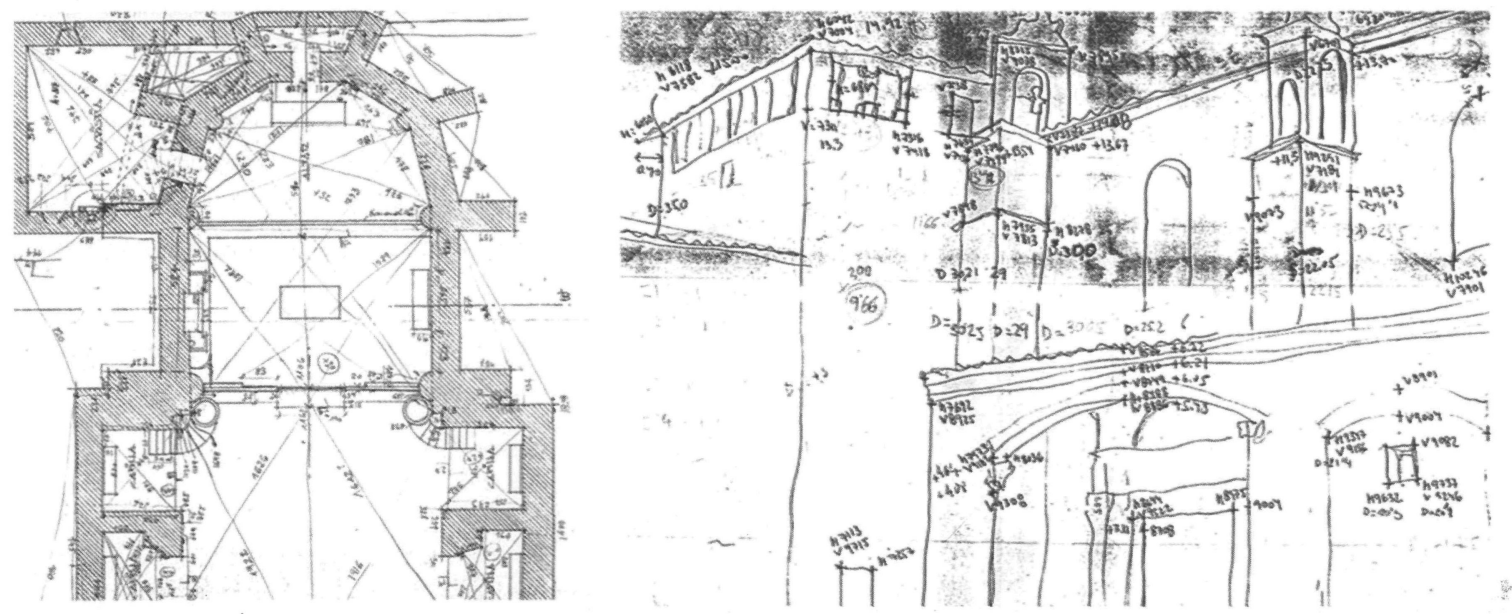

Dos croquis distintos: utilizando cinta métrica y anotando ángulos verticales y horizontales leidos con un taquimetro.

topográficos, a base de instrumentos, como el nivek(que sólo nos permite medir ángulos horizontales, aunque también diferencias de cotas, por medio de la típica regla de aluminio) y el taquímetro o teodolito (con el que podemos medir ángulos horizontales y verticales).

Recientemente ha aparecido la llamada estación total, taquímetro que permite capturar los datos digitalmente $\mathrm{e}$ introducirlos en un ordenador para su posterior manipulación con programas de CAD. Si este instrumento se complementa con un distanciómetro ${ }^{12}$, basta prácticamente hacer una "estación" (un punto desde donde se realizan las lecturas y mediciones) para poder tener situados, espacialmente, todos los puntos que vemos y queremos "levantar".

\section{3.b) RESTITUCIONESYREPRESENTACIONES}

Además de las técnicas de restitución fotogramétrica a partir de pares estereoscópicos, ya reseñadas en párrafos anteriores, la información geométrica medible que puede

(c) Consejo Superior de Investigaciones Científicas

Licencia Creative Commons 3.0 España (by-nc) suministrar una fotografia aislada es siempre asombrosa, basta conocer ligeramente las leyes de la perspectiva. Teniendo algunos "puntos de apoyo" (puntos de los que conocemos su posición real), existen técnicas para ortogonalizar fotografías, es decir, para corregir las deformaciones perspectivas, introduciendo deformaciones opuestas, muy útil en el caso de la representación de elementos fundamentalmente planos, como fachadas de un edificio, por ejemplo.

El dibujo mediante ordenador representa, en los últimos años, uno de los medios de representación más extendidos -y ya casi obligatorios- en la oficina del proyectista. La facilidad con la que un dibujo o parte del mismo puede ser copiada, deformada, cambiada de escala, etc., permite hacermás asequibles las labores de restitución, el encaje sobre una geometría coherente de todos los datos que se tomaron previamente. El dibujo en tres dimensiones (3D) rara vez presenta utilidad adicional, salvo para cuando se preparan representaciones virtuales o modelos volumétricos ("renderizaciones").

http://informesdelaconstruccion.revistas.csic.es 

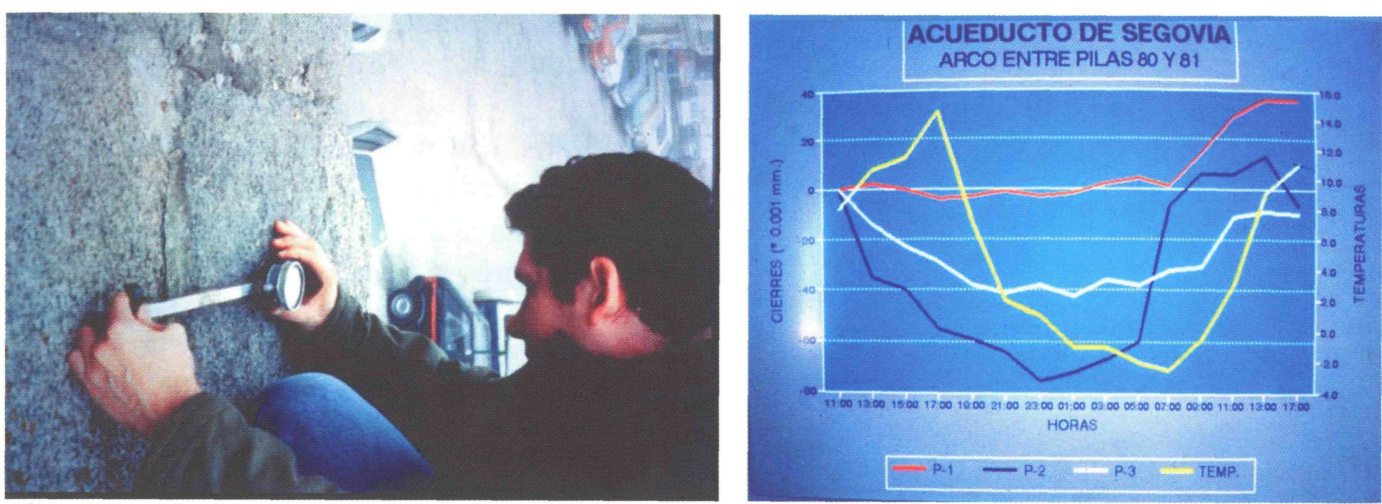

Utilizando extensómetros mecánicos y sondas térmicas se pudo detectar en el acueducto de Segovia la acomodación de los sillares, en el sentido longitudinal de la construcción, ante los cambios de temperatura exteriores.

Acoplando extensómetros a cintas metálicas, se pueden realizar medidas de convergencia, es decir, comprobaciones, con precisiones de fracciones de milímetro, de distancias entre 1 y 30 metros.

Para medir desplomes o inclinaciones pueden utilizarse péndulos inversos, dianas con visores aplomados o clinómetros de precisión; estos últimos, incluso conectados con un sistema de almacenamiento informático.

El inclinómetro, sin embargo, es una especie de "topo" que puede ser introducido en perforaciones entubadas de pequeño diámetro efectuadas en el terreno, pudiéndose detectar variaciones en la verticalidad y, por tanto, desplazamientos relativos de capas del terreno entre sí.

\section{2.b) CONTROL DE VIBRACIONES}

Para el control de vibraciones es necesario colocar acelerómetros o sensores direccionales en los puntos donde se quiere detectar el grado de vibración, la cual puede ser capturada en osciloscopios donde la onda puede ser procesada en función del período y amplitud que posea. La fuente de producción de ondas puede ser natural (viento, acción sísmica, tráfico de vehículos...) o bien artificial (golpes, vibración normalizada...), para cotejar los valores que se obtienen en distintos casos o las posibles resonancias.

\section{2.c) CONTROL DE TENSIONES}

Realmente no se pueden medir tensiones si no es de un modo indirecto, es decir, controlando las deformaciones que producen. En este sentido, todas las técnicas de utilización de gatos planos ("flat jacks") pasan por el uso conjunto de extensometrías ${ }^{11}$. Del mismo modo, la colocación de células de carga, habituales en cabezas de anclaje de postensados, se basan en la medida de la variación de la resistividad eléctrica que tienen pequeñas resistencias ante deformaciones longitudinales.

\section{2.d) OTROSCONTROLES}

Evidentemente, todos los controles de temperatura, presión, humedad, etc., pueden ser también automatizados y almacenados periódicamente en soporte informático, para su estudio y posterior seguimiento.

Queda claro, para cualquier lector especializado, que nos dejamos muchos instrumentos sin mencionar, puesto que los laboratorios de control e instrumentación poseen, además de los mencionados, gran variedad de instrumentos especializados en medidas de alta precisión, pero hemos mencionado aquéllos que, alguna vez, hemos tenido la ocasión de utilizar.

\section{LAREALIZACIÓN DEL PROYECTO}

Decimos realización y no redacción con una clara intención: abarcar también los trabajos que hay que realizar, antes de hacer propiamente la redacción del proyecto.

En este sentido, vamos a hablar de tecnologías al alcance de casi todos los estudios de arquitectura hoy en día, con la clasificación utilizada en la proyectación.

\section{3.a) TOMADEDATOS GEOMÉTRICA}

La toma de datos es una de las fases fundamentales en el conocimiento de cualquier construcción cuya restauración va a ser abordada. Por supuesto, en ella quedaría incluida la fase de estudios previos, si éstos se realizan. En concreto, la toma de datos geométricos es siempre una de las labores que ayuda a entender, de un modo más directo, el objeto de la restauración. Para ello, la cinta métrica ha sido siempre un instrumento común e imprescindible, las medidas trianguladas, a ser posible con redundancias que nos permitan verificar errores, son la base más sólida para una fiel representación en planta.

Estas medidas pueden hacerse sin grandes conocimientos 


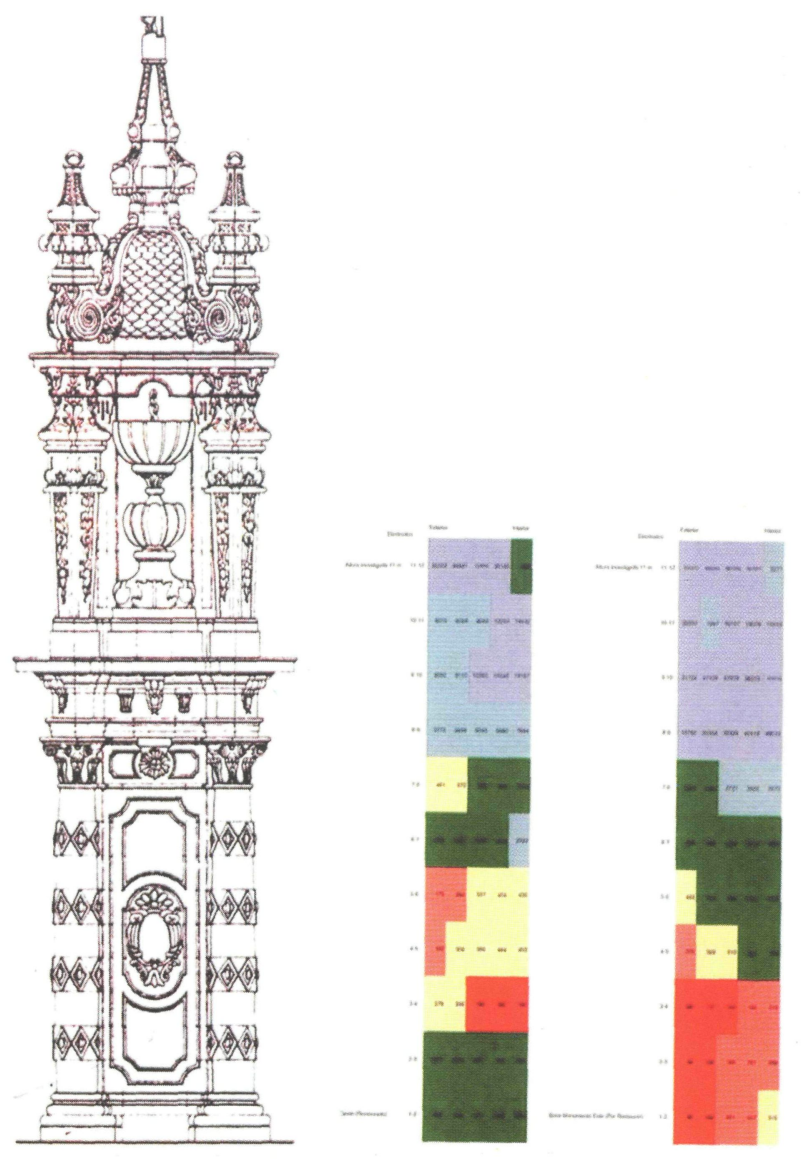

Mediante la resistividad eléctrica se detectó que el método de limpieza sobre un grupo escultórico estaba concentrando agua en el interior, al comparar la humedad creciente hacia abajo en el grupo sin restaurar con la "bolsa" generada en el restaurado.

\section{LAMONITORIZACIÓN}

Por monitorización entendemos el conjunto de medidas que pueden realizarse para controlar un proceso que varía, o puede variar, con el tiempo. En este caso, refiriéndonos a una restauración, nos interesará controlar aspectos relacionados con movimientos o deformaciones de la cons- trucción o variables que puedan incidir en su conservación (temperatura, grado de humedad, etc.)

Las labores de monitorización pueden ser clasificadas con distintos criterios. Con el convencimiento de que no vamos a ser exhaustivos, ya que sólo pretendemos reflejar aquellos procedimientos que alguna vez hemos utilizado, pasamoos a ofrecer la siguiente clasificación.

\section{2.a) CONTROL DE DEFORMACIONES}

Mediante topografía de precisión con correcciones, utilizando algoritmos matemáticos, puede llegarse a precisión de milímetros, midiendo a distancias de unos 100 metros. Con instrumentos más sofisticados, como el mecómeter, la precisión es aún mayor ${ }^{10}$, pero es necesario personal altamente especializado para su manejo, pues hasta el grado de humedad del aire influye en la medición. En todos estos casos suele combinarse la medición angular (triangulaciones) con la interferometría de rayo láser, que permite la medida de distancias directamente, mediante reflexión del haz de luz en un prisma. Todas estas técnicas pueden ser útiles cuando se pretende medir desplomes, hundimientos o cualquier tipo de movimientos globales de paramentos o paños de una construcción.

En pequeñas longitudes (hsasta unos $60 \mathrm{~cm}$ ) se pueden manejar instrumentos de medida más precisos (hasta la milésima de milímetro), ya sea manualmente, con extensómetros, ya sea de un modo automático, con cuerda vibrante, por ejemplo, enviando periódicamente lecturas a un ordenador. Estas técnicas son imprescindibles si se quieren medir las variaciones en la apertura de fisuras o movimientos relativos entre dos elementos constructivos.

Los fleximetros o "relojes", habitualmente utilizados para medir flechas en pruebas de carga, pueden ser instrumentos adecuados para comprobar deformaciones en pequeños períodos de tiempo.

\section{CLASIFICACIÓN DELOS TRABAJOS DE MONITORIZACIÓN}

\begin{tabular}{|lll|}
\hline 2.a) CONTROL DE DEFORMACIONES & Gran longitud & $\begin{array}{l}\text { Mecómeter } \\
\text { Topografía de precisión } \\
\text { Medidas de convergencia } \\
\text { Extensómetro } \\
\text { Cuerda vibrante }\end{array}$ \\
& $\begin{array}{l}\text { Longitud media } \\
\text { Pequeña longitud }\end{array}$ & $\begin{array}{l}\text { Inclinómetro } \\
\text { Clinómetro } \\
\text { Péndulos, dianas, etc. }\end{array}$ \\
\hline & $\begin{array}{l}\text { Fesplazetros } \\
\text { Medidas de inclinaciones }\end{array}$ & \\
\hline 2.b) CONTROL DE VIBRACIONES & Acelerómetros y osciloscopios \\
\hline & & \\
\hline 2.c) CONTROL DE TENSIONES & Células de carga \\
& Gatos planos \\
\hline
\end{tabular}




\section{1.d) ENSAYOSDEMATERIALES}

En muchos casos podemos acudir a laboratorios que pueden identificarnos determinados materiales o componentes utilizando distintas técnicas: lámina delgada, espectrografía de rayos $\mathrm{X}$, análisis químicos, etc.

Pero mi experiencia particular ha obtenido más conclusiones a partir de los ensayos mecánicos, habitualmente la tensión de rotura del material ante determinado tipo de esfuerzo, lo cual nos ha permitido estar seguros de la estabilidad de una estructura, al cotejar los valores de rotura con la situación de servicio (situación resultado de análisis sobre modelos de cálculo, más o menos ajustados).

Los ensayos de comportamiento ante determinados productos químicos o tratamientos son también fundamentales si queremos estar seguros de que no va ser peor el remedio que la enfermedad. Para ello disponemos de la posibilidad de hacer ensayos de envejecimiento acelerado, verificación de posibles variaciones de comportamientos higrotérmicos, mecánicos o, sencillamente, de aspecto externo, etc., toda una serie de técnicas que, en muchos casos con costes razonables, pueden proporcionarnos laboratorios especializados.

De todos modos, suele ser más concluyente el conocimiento de experiencias o tratamientos similares que lleven mucho tiempo sometidos a condiciones similares a nuestro caso $^{6}$.

\section{1.e) INVESTIGACIONESINSITU}

La realización de catas es muchas veces el único método para conocer un aspecto constructivo o un síntoma patológico. Cuando llegamos a contar con la colaboración de arqueólogos, el conocimiento constructivo se ve sensi- blemente ampliado en su vertiente histórica. Cuando el estudio es geotécnico, suele atenderse más a problemas de estabilidad afectados por el subsuelo ${ }^{7}$.

Para mí ha sido de mucho interés la utilización de métodos geofísicos no destructivos para auscultar el interior de construcciones antiguas, no solamente a efectos de detectar oquedades o cambios de composición, sino también para detectar concentraciones de humedad, o la calidad y estado de deterioro del material.

Sé de la utilización del geo-rádar para detectar estructuras ocultas sin levantar el suelo, para lo que también se utiliza las medidas de resistividad eléctrica. Nosotros, sin embargo, hemos utilizado este método para detectar la compacidad interior de un muro, el contenido de humedad e, incluso, el grado de contaminación superficial que posee la fábrica ${ }^{8}$.

La medida de vibraciones mediante colocación de acelerómetros es un método clásico para determinar el grado de afección ante vibraciones del tráfico o acciones dinámicas, como el viento o el sismo. Sin embargo, la auscultación dinámica la hemos utilizado para determinar el grado de conectividad de los elementos de una fábrica y, al mismo tiempo, para obtener el módulo de elasticidad de la misma, con resultados altamente satisfactorios ${ }^{9}$.

Por último, la medida de velocidad de transmisión de ultrasonidos, se ha revelado como el mejor método para determinar la calidad del granito, de modo que es el primer factor que se anota en la base de datos que estamos realizando, sillar por sillar, en la restauración del acueducto romano de Segovia. Dicha velocidad nos permite realizar un seguimiento objetivo del grado de meteorización del granito y también nos puede indicar la oportunidad de consolidar o no.
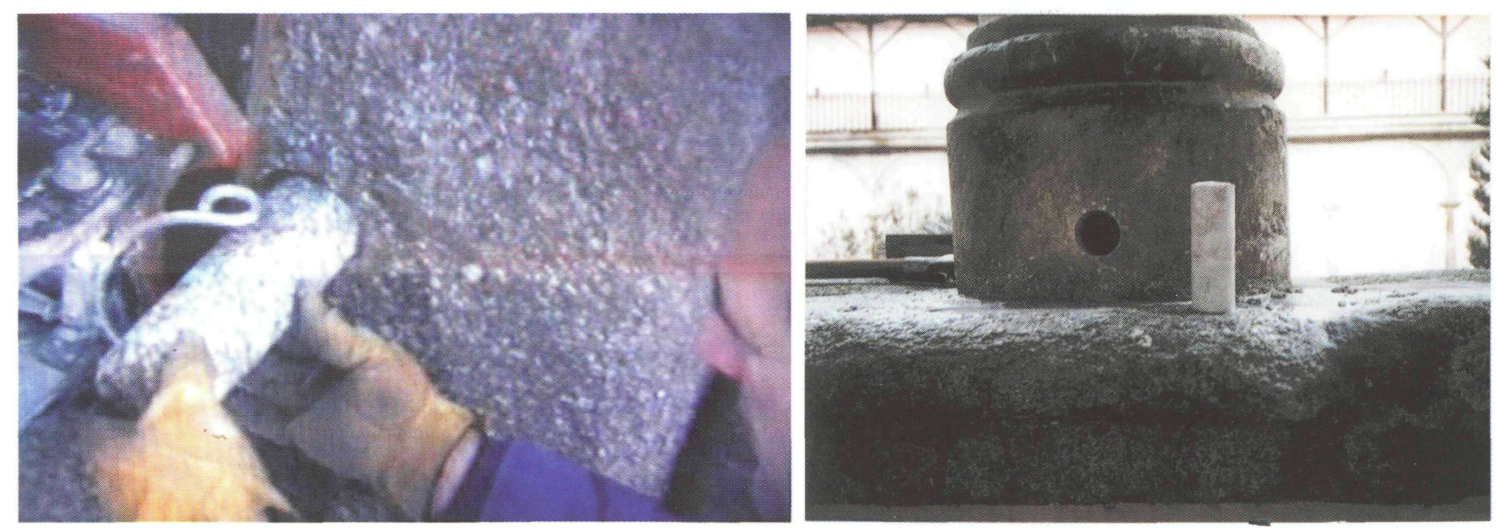

Obtención de testigos en los sillares graniticos del acueducto de Segovia para su estudio y comparación con el extraido de canteras similares. A la derecha, testigos de las columnas de mármol de un claustro para determinar su seguridad ante las cargas soportadas. 


\section{1.b) ESTUDIOSAMBIENTALES}

Los estudios ambientales tienen más relevancia en la medida en que se hayan venido desarrollando durante más tiempo, de modo que la comparación entre distintas situaciones y momentos es la que puede determinar las variables significativas.

Nuestra experiencia en este apartado se circunscribe, casi exclusivamente, a los estudios realizados para el ya mencionado acueducto de Segovia, con el problema de que, hasta el comienzo de nuestros trabajos, no se habían realizado estudios con los que pudiésemos comparar. De hecho, los estudios microbiológicos ${ }^{4}$ arrojaron una cantidad anormal de microorganismos sobre la superficie de la piedra; sin embargo, aún está por comprobarse el efecto biocida de la restauración actual y hasta qué punto se volverá a la situación anterior. Sin embargo, en lo que respecta a las medidas que se pudieron realizar sobre niveles atmosféricos de contaminación, se pudo comprobar cómo, una vez cortado el tráfico bajo el acueducto, seguían existiendo importantes variaciones debidas a la puesta en marcha de las calefacciones o a la masiva influencia de vehículos en las cercanías de la construcción romana en días festivos.

\section{1.c) ESTUDIOSGEOMÉTRICOS}

La determinación de la geometría de la construcción que estamos estudiando, el llamado levantamiento (se entiende que de los planos del edificio), es uno de los pilares fundamentales en los que se basa el conocimiento del mismo, así como la interpretación constructiva e incluso histórica, evolutivamente hablando.

Con la palabra topografia hemos querido incluir desde las medidas directas con cinta métrica hasta la utilización de niveles, taquímetros o lo que hoy en día se llama estación total.

En el apartado fotogrametría incluimos todo tipo de lectura o medida geométrica realizada a partir de cualquier clase de fotografia: medidas directas en fotografías más o menos frontales, medidas en fotos con perspectivas acusadas, utilizando proporciones o series matemáticas simples, fotogrametrías aéreas existentes, habitualmente, sobre el territorio y las poblaciones, restituciones fotogramétricas a partir de pares estereoscópicos realizados ad hoc, ya sean en dibujos planos o en tres dimensiones sobre formato electrónico (habitualmente llamado $\mathrm{CAD}$ ).
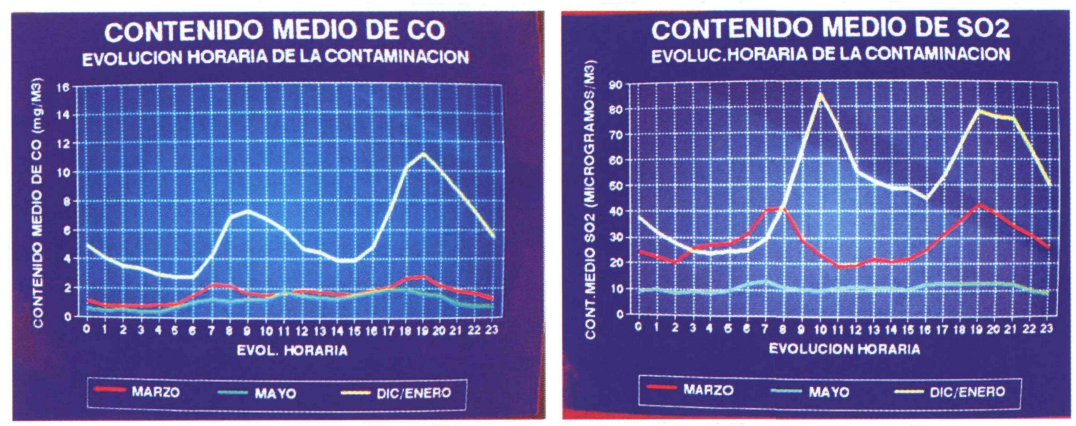

Algunas curvas de niveles de contaminación estudiadas en los estudios previos del acueducto de Segovia.

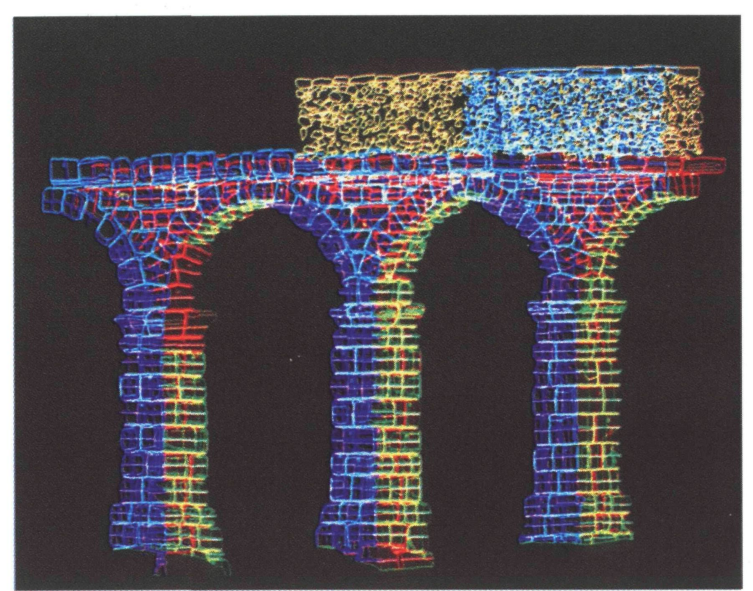

Fotogrametría en 3D realizada para el acueducto de Segovia. (c) Consejo Superior de Investigaciones Científicas Licencia Creative Commons 3.0 España (by-nc)
Aquí es donde la tecnología nos ha proporcionado más avances, pero también más pérdida de control por parte del futuro autor del proyecto de restauración, el más interesado en el conocimiento directo de la construcción. No olvidemos nunca que la fotogrametría mide sólo aquello que puede fotografiarse (en muchos casos lo más frontalmente posible) y que la verdadera información de la geometría que necesitamos es, muchas veces, la interior, aquélla que no se ve, la que hay que obtener cotejando y comparando con otras medidas, metiéndose por huecos inverosímiles, llenándose de polvo y suciedad en excursiones a las "tripas" del edificio, o jugándose el físico para llegar lo más cerca posible te los sitios inaccesibles... Pero la recompensa nos espera ahí: todas las medidas llegan a casary, de súbito, entendemos gruesos de muros, huecos tapados y desplomes de paramentos ${ }^{5}$.

http://informesdelaconstruccion.revistas.csic.es 


\section{A MODODE CONCLUSIÓN}

Como las antiguas fábulas con sus moralejas, no hemos podido resistirnos a la tentación de exponer unas reflexiones finales, reflexiones que también pueden quedar como propuestas lanzadas al aire para quien proceda o las quiera recoger.

Si de lo que se trata es de conservar, utilicemos la técnica para minimizar la intervención y no -como ocurre con demasiada frecuencia- para tratar de justificar restauraciones más duras, más destructivas, menos conservadoras.

\section{NOTAS}

${ }^{1}$ Siempre nos hemos hecho esta reflexión cuando algún comentario historicista apuntaba a que tal o cual solución no podía ser de una época determinada, porque los habitantes de la misma ya conocían una técnica más avanzada... En efecto, hoy existe la tecnología para viajar a la Luna, pero ¿ cuántas personas pueden ir allí en realidad?

${ }^{2}$ Evidentemente esta clasificación es muy subjetiva y está hecha desde el punto de vista de un técnico como el que suscribe, técnico que habitualmente sólo dispone personalmente de la tecnología del tercer apartado, pero, al fin y al cabo, ¿ no es todo artículo algo totalmente subjetivo?

${ }^{3}$ Respecto a todos los trabajos realizados en el acueducto de Segovia, citados repetidamente en este artículo, pueden consultarse, entre otras, las siguientes publicaciones:

"El acueducto de Segovia". Informes de la Construcción. Número 437. Madrid, mayo-junio 1995. pp. 5 a 31.

"Roman aqueduct of Segovia". Structural analysis of historical constructions. Possibilities of numerical and experimental techniques. Barcelona, 1997, pp. 307 a 340.

${ }^{4}$ Realizados por el profesor Fernando Laborda, de la universidad de Alcalá de Henares.

${ }^{5}$ Como dice mi querido amigo, el arquitecto Ignacio Gárate, al edificio hay que magrearlo, como si de tu pareja se tratase, porque suele ser retraído y le cuesta mucho soltar su información.

${ }^{6}$ La utilización de la misma resina epoxídica para sellar fisuras en los sillares graníticos que la que fue utilizada hace más de 25 años en otra restauración del acueducto romano de Segovia, ha sido sancionada por la bondad del comportamiento ante la intemperie en todos estos años, garantía que no podía dar ningún ensayo de envejecimiento acelerado actual.

\footnotetext{
${ }^{7}$ No hay que escatimar precauciones ni dosis de prudencia en la interpretación de muchos estudios geotécnicos que, enarbolando la bandera de la seguridad estructural, suelen realizar recomendaciones de recalces innecesarios y, en muchos casos, nefastos. Es éste un tema preocupante, ya que la filosofia habitual de este tipo de estudios está referida a nuevas construcciones y no a edificios que llevan varios siglos en situación estable.
}

El ordenador no es más que una calculadora, que hace lo que su usuario es capaz de resolver por sí mismo ${ }^{17}$.

Para fomentar el uso ingenioso de la tecnología, los técnicos responsables de una restauración deberían percibir sus honorarios proporcionalmente al dinero que han ahorrado y no a la cuantía de presupuesto invertida ${ }^{18}$, ya que, con mucho presupuesto, cualquiera puede encontrar una solución.

Tan malo es pecar de omisión como de exceso, por lo que la mejor técnica será siempre la más precisa, la justa y necesaria.

${ }^{8}$ La medida de la resistividad eléctrica superficial permite, dado su gran rango de variaciones, detectar cambios muy sutiles en las fábricas, siempre que no existan elementos conductores (trozos metálicos, por ejemplo) que enmascaren los resultados. Concretamente, realizando unas pruebas sobre los sillares de granito del acueducto de Segovia, se comprobó que el método servía para discriminar perfectamente los sillares con suciedad superficial por contaminación de los ya limpiados, dada la conductividad de las sales contenidas en la capa externa, con lo que se utiliza este método para determinar, en el futuro, de un modo objetivo, el grado de ensuciamiento debido a la contaminación atmosférica.

${ }^{9}$ Este método no deja de ser una sofisticación respecto al clásico e intuitivo golpe sobre una pared, o zapatazo sobre una bóveda, para comprobar lo "suelta" o rígida que está.

${ }^{10}$ Una vez llegué a asistir a una demostración de cómo se medía la deformación de una presa, según se iba llenando de agua, a través de medidas con el mecómeter desde la propia presa a puntos fijos en las montañas colindantes.

"La técnica del gato plano se basa en medir la distancia vertical, p. e., entre dos puntos de un paramento. Se corta localmente el material existente entre ambos puntos y se mide de nuevo la distancia entre los dos puntos anteriores, que se habrá acortado. Se introduce después un gato plano en el corte y se da tensión hasta que la distancia original vuelve a ser la misma. Teóricamente, la fuerza que el gato plano aporta a la superficie del corte puede determinar la tensión a la que estaba trabajando el paramento.

12 Últimamente han aparecido en el mercado distanciómetros mediante haz visible de láser que no necesitan prisma para poderla reflejar, a fin de medir la distancia por interferometría. Su utilidad resulta obvia cuando se quieren dibujar partes de una construcción a las que no podemos acceder.

${ }^{13}$ En arqueología, la realización de modelos virtuales con ordenador puede ayudar a recrear, de un modo casi real, los espacios que ahora no pueden ser reconstruidos, basándose científicamente en los restos hallados.

${ }^{14}$ Hay que llamar aquí la intención sobre la excesiva "fe" que a veces se tiene sobre los resultados obtenidos con un ordenador, sin que el modelo matemático esté suficientemente calibrado; lapalabra inglesa reliability refleja esta confianza sobre el modelo. 


\begin{abstract}
${ }^{15}$ Evidentemente, nos estamos limitando en la clasificación a las obras de restauración desde el punto de vista arquitectónico. La restauración de bienes muebles: pintura mural, maderas, telas, etc., tiene sus propias técnicas y sus propios ensayos auxiliares.

${ }^{16}$ Casi es obligado citar los estudios realizados por nuestros compañeros: Ignacio Gárate, en el campo de los revestimientos de cal y yeso, Enrique Nuere, sobre carpintería de madera y lacería, Antonio Almagro, en el campo de la restitución fotogramétrica e Ignacio Paricio, sobre los métodos constructivos a lo largo de la historia, etc., arquitectos que han dedicado, de uno u otro modo, su vida profesional a la conservación o el estudio de edificios históricos.
\end{abstract}

${ }^{17}$ Viene muy a propósito la acertada cita del comienzo del manual del conocido programa de elementos finitos SAP, que dice textualmente: "An incapable engineer cannot do with a ton of computer output what a good engineer can do on the back of an envelope". Incluso más adelante, el manual se permite bromear con el significado de SAP en "slang" o argot, que significa bobo, recordando que el uso de todo programa de ordenador requiere inteligencia.

${ }^{18} \mathrm{Creo}$ que en China los médicos cobraban honorarios mientras sus pacientes estaban sanos, dejando de percibirlos al tener éstos alguna enfermedad. Interesante método, que muestra cierto paralelismo con el conservador responsable de un edificio histórico.

\title{
Publicación del Instituto Eduardo Torroja - CSIC
}

\author{
Número monográfico de INFORMES
}

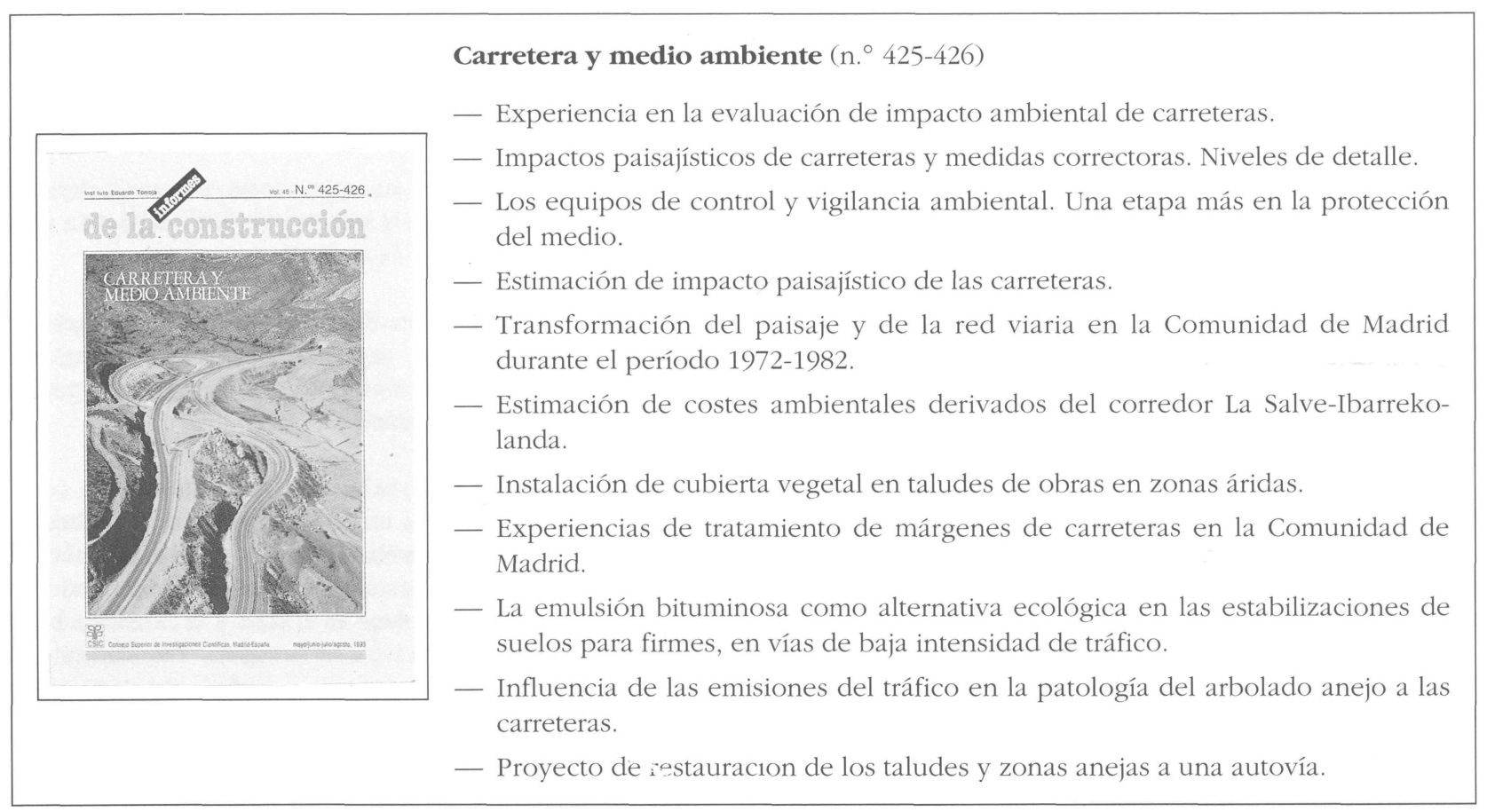

